\title{
Simultaneous Determination of Twenty-Two Components in Asari Radix et Rhizoma by Ultra Performance Liquid Chromatography Coupled with Quadrupole Time-of-Flight Mass Spectrometry
}

Authors

Affiliation
Hao Wen, Hui-yuan Gao, Wen Qi, Feng Xiao, Li-li Wang, Dong Wang, Dan Yuan

Department of Traditional Chinese Medicine, Shenyang Pharmaceutical University, Shenyang, China
Key words

- Asari Radix et Rhizoma

- Aristolochiaceae

- lignans

- essential oils

- aristolochic acids

- UHPLC-QTOF/MS received June 21,2014

revised October 14, 2014

accepted October 17, 2014

Bibliography

DOI http://dx.doi.org/

10.1055/s-0034-1383296

Planta Med 2014; 80:

1753-1762 @ Georg Thieme

Verlag KG Stuttgart · New York .

ISSN 0032-0943

Correspondence

Prof. Dr. Dan Yuan

Department of Traditional

Chinese Medicine

Shenyang Pharmaceutical

University

103 Wenhua Road

Shenyang 110016

China

Phone: + 8602423986502

Fax: +8602423986502

yuandan_kampo@163.com

\section{Abstract}

$\nabla$

Asari Radix et Rhizoma is a herbal medicine for the treatment of common cold, rhinitis, etc. An ultra performance liquid chromatography coupled with quadrupole time-of-flight mass spectrometry method has been established for the rapid analysis of 22 components in 27 samples from the raw materials of Asari Radix et Rhizoma and an adulterant. A total of 5 lignans, 5 essential oils, 3 aristolochic acids, 4 alkamides, and 5 flavanoids were identified by co-chromatography of samples extracts and comparison of the retention time, UV spectra, characteristic molecular ions, and fragment ions with those of authentic standards, or tentatively identified by MS/MS determination along with MassFragment software. Moreover, the method was validated for the simultaneous quantification and semi-quantification of 20 components. The samples from Asarum heterotropoides var. mandshuricum differed in the quantity of 2-methoxyl-4,5-methylenedioxypropiophenone and kakuol from those of Asarum sieboldii var. seoulense, and the chemical difference was supported by principal component analysis and orthogonal partial least squared discriminant analysis based on dataset obtained from UHPLCQTOF/MS. In comparison with the samples from the two medicinal Asarum species mentioned above, those from $A$. himalaicum differed in the quality and quantity of major compounds and contained higher amounts of aristolochic acid I.

\section{Introduction}

$\nabla$

Asari Radix et Rhizoma (ARR, Xixin in Chinese) is a well known herbal medicine which has been mainly used to treat common cold, influenza, rhinitis, or as a local anesthetic agent, etc [1,2]. According to the Chinese Pharmacopoeia [3], it is derived botanically from the dry roots and rhi-

\section{Abbreviations}

$\nabla$

AA: $\quad$ aristolochic acid

AAN: aristolochic acid nephropathy

ACN: acetonitrile

AL-I: aristololactam I

APCI: atmospheric pressure chemical ionization

ARR: $\quad$ Asari Radix et Rhizoma

DAD: diode-array detector

ESI: electrospray ionization

FWHM: full width at half maximum

GAP: good agricultural practice

LOD: limit of detection

LOQ: limit of quantitation

OPLS-DA: orthogonal partial least squared discriminant analysis

PCA: principal component analysis

PTFE: polyterafluoroethylene

QTOF/MS: quadrupole time-of-flight mass spectrometry

RSD: relative standard deviations

UHPLC: ultra high performance liquid chromatography

XIC: extracted ion chromatogram

Supporting information available online at http://www.thieme-connect.de/products 
and should be responsible for the aforementioned activities [11]. Among the three Asarum spp. recorded under the same monograph of ARR in the Chinese Pharmacopoeias, the raw materials from $A$. heterotropoides var. mandshuricum and A. sieboldii var. seoulense are called "Liaoxixin" in China, and both Asarum species are mixed in most herbal farms in northeastern China. The herbal materials from $A$. sieboldii have been rarely commercially available due to its depleted wild populations [12,13]. Meanwhile, there are many adulterants of ARR in China, such as the roots and rhizomes of $A$. himalaicum Hook. f. et Thoms. ex. Klotzsch., A. caulescens Maxim., A. maximum Hemsl., and A. forbesii Maxim. [14]. Among them, the roots and rhizomes of $A$. himalaicum have been used as a folk medicine and are commercially available in a relatively large amount in some regions of western China [15]. However, there have been still no scientific studies to characterize the rationality of the traditional practice about ARR and its adulterants until now, which is a serious problem with regard to their efficacy, quality control, and safety [16].

According to the Chinese Pharmacopoeia [3], the essential oil and asarinin are used as marker compounds for the quality control of ARR. Several holistic chemical profiling methods of ARR have been reported, such as quantification of 2-3 lignans and 1-2 constituents of essential oils using (HPLC)-UV [17-19]. It is worth noting that ARR contains AAs, some of which can cause AAN. The US FDA has prohibited the import of the herbal preparations in which the herbal medicines containing AAs are formulated [20]. Because ARR contains AAs in extremely small amount, an acceptable limit for AAs in ARR has been established in the Chinese and Japanese Pharmacopoeias. In the limit tests by HPLC method, the content of AA-I in dry materials of ARR must be less than $0.001 \%$ (10 ppm) according to the Chinese Pharmacopoeia [3], and the sample solution of ARR must show no peak at the retention time corresponding to AA-I from the standard solution in the Japanese Pharmacopoeia [21]. Although AA-I in ARR was determined by HPLC-UV and LC-APCI-MS/MS methods [22-24], few papers investigated the content of other AA compounds in ARR, such as AA-IVa and AL-I, in relation to AAN [25-27]. Thus, it is necessary to develop a method for simultaneous determination of active or marker components and AAs in ARR.

Generally, the use of conventional HPLC methods is difficult and time-consuming for simultaneous determination of lignans, essential oils, flavanoids, AAs, and alkamides in ARR because of their relative low efficient stationary phases. Recently, the use of UHPLC coupled with QTOF/MS has been an effective approach for rapid analysis of multi-components of herbal medicines due to its higher peak capacity, greater resolution, increased sensitivity, and rich data on accurate molecular formulae for structural identification of unknown compounds $[28,29]$. Since many unknown components may be present in ARR, most of which are new or without reference standards, using TOF/MS to identify them is important based on the molecular formula and fragmentation of the known components.

In the present study, a multi-component quantification fingerprinting approach based on UHPLC-QTOF/MS techniques has been developed, which combined the chemical profiling and semi-quantification of over 20 components in the roots and rhizomes of $A$. heterotropoides var. mandshuricum and $A$. sieboldii var. seoulense and the roots of $A$. himalaicum. This is the first report on the comprehensive evaluation of the chemical profile of the roots and rhizomes of $A$. heterotropoides var. mandshuricum and $A$. sieboldii var. seoulense and the roots of $A$. himalaicum by UHPLC-QTOF/MS method.

\section{Results and Discussion}

$\nabla$

In order to obtain satisfactory extraction efficiency for all the analytes, extractive methods (ultrasonic and refluxing), solvents (50\%, 75\%, and 100\% methanol), and time (30 and $60 \mathrm{~min}$ ) were assessed based on single factor experiments. The best extraction efficiency was obtained by refluxing extraction with $100 \%$ methanol for $30 \mathrm{~min}$.

The methanolic extracts of $A$. heterotropoides var. mandshuricum (sample \#3) and A. sieboldii var. seoulense (sample \#10) were used for the optimization of UHPLC conditions; their representative UHPLC-UV and UHPLC-MS chromatograms are presented in - Fig. 1. Using the optimal gradient elution as described in Material and Methods, all the specific peaks were satisfactorily separated within $22 \mathrm{~min}$.

In order to characterize the chemical composition, the methanolic extracts of the root and rhizome of $A$. heterotropoides var. mandshuricum (sample \#3) and A. sieboldii var. seoulense (sample \#10) were subjected to UHPLC-QTOF/MS analysis. Twenty-two specific peaks (labeled peaks 1-22, O Fig. 1) in the UHPLC chromatograms were characterized by UV absorptions obtained with Waters DAD. By co-chromatography and comparison of the retention time, UV spectra, and characteristic molecular ions and fragment ions with the authentic standards, compounds corresponding to 17 peaks were identified ( $\odot$ Table $\mathbf{1}$ ).

Due to absence of reference compounds, the compounds corresponding to the rest 5 compounds were tentatively identified by MS/MS determination along with Waters MassFragment software, UV spectra, and comparison with literature data ( Fig. 1 and Table 1). Peak $5\left(t_{R} 3.95 \mathrm{~min}\right)$ generated the positive molecular ion at $m / z 597.1819[\mathrm{M}+\mathrm{H}]^{+}$, corresponding to the molecular formula $\mathrm{C}_{27} \mathrm{H}_{32} \mathrm{O}_{15}$, and two fragment ions at $\mathrm{m} / \mathrm{z} 435.1291$ (loss of a hexosyl, 162 mass units) and 273.0763 (loss of two hexosyls, 324 mass units), which were the same as those of ( $2 R)$-naringenin 5,7-di-O-glucoside (peak $1, t_{R} 1.78 \mathrm{~min}$ ) in the MS chromatograms. The UV spectra of peak $\mathbf{5}(288,323 \mathrm{~nm})$ was also similar to that of (2R)-naringenin 5,7-di-O-glucoside $(277,320 \mathrm{~nm})$, suggesting that it should be a flavanoid. Thus, the compound corresponding to peak $\mathbf{5}$ was tentatively identified as the isomer of (2R)-naringenin 5,7-di-O-glucoside. According to the UV spectra of peak $\mathbf{5}$, the compound corresponding to peak $\mathbf{5}$ may be $(2 R)$ naringenin 4',7-di- 0 -hexoside [30].

Peaks $7\left(t_{R} 6.53 \mathrm{~min}\right)$ and $9\left(t_{R} 7.52 \mathrm{~min}\right)$ generated the $[\mathrm{M}+\mathrm{H}]^{+}$ ions at $m / z$ 183.1021, corresponding to the same molecular formula $\mathrm{C}_{10} \mathrm{H}_{14} \mathrm{O}_{3}$. High collision energy resulted in two important fragment ions, at $m / z 168.0779$ (loss of a methyl, 15 mass units) and 153.0548 (loss of two methyls, 30 mass units). These fragment ions are in accordance with the fragmentation pathways of 3,4,5-trimethoxytoluene and 2,4,6-trimethoxytoluene shown in the phytochemical study on ARR [31]. According to the difference of polarity [32], peaks 7 and 9 were tentatively identified as 3,4,5trimethoxytoluene and 2,4,6-trimethoxytoluene, respectively.

Peak $22\left(t_{R} 21.48 \mathrm{~min}\right)$ showed the similar UV absorption at 235 and $259 \mathrm{~nm}$ and generated the same $[\mathrm{M}+\mathrm{H}]^{+}$ion at $\mathrm{m} / \mathrm{z}$ 248.2014, corresponding to the molecular formula $\mathrm{C}_{16} \mathrm{H}_{25} \mathrm{NO}$. High collision energy resulted in diagnostic fragment ion at $\mathrm{m} / \mathrm{z}$ 167.1310 (loss of $\mathrm{C}_{6} \mathrm{H}_{9}, 81$ mass units) and $m / z 152.1075$ (loss of $\mathrm{C}_{7} \mathrm{H}_{12}, 95$ mass units). The fragment ion was shown in the fragmentation pathways of a pair of isomers, $N$-isobutyl$2 E, 4 E, 8 Z, 10 Z$-dodecatetraenamide and $N$-isobutyl-2E,4E,8Z,10Edodecatetraenamide in the studies by Yasuda et al. [10] and Luo et al. [33]. So the compound corresponding to peak 22 was tenta- 


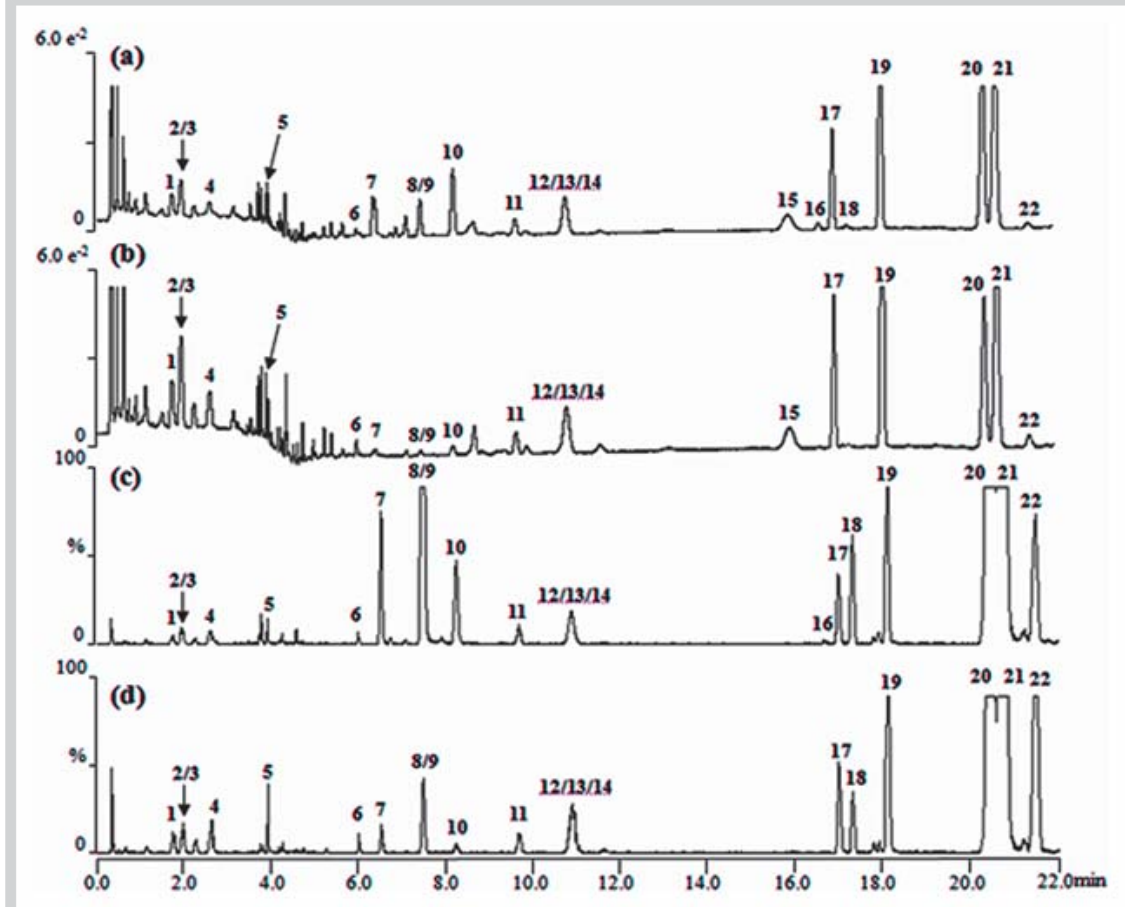

Fig. 1 Representative UHPLC-UV ( $\mathbf{a}$ and $\mathbf{b}$, detected at $287 \mathrm{~nm}$ ) and extracted ion chromatograms ( $\mathbf{c}$ and $\mathbf{d}$, base peak intensity in positive ionization mode) of the extracts of the roots of $A$. heterotropoides var. mandshuricum (sample \#3, a and c) and $A$. sieboldii var. seoulense (sample \#10, $\mathbf{b}$ and $\mathbf{d}$ ). See $\odot$ Table 1 for the peak numbers, and see Materials and Methods for UHPLC-QTOF/MS conditions. (Color figure available online only.)

Table 1 Marker compounds identified from A. heterotropoides var. mandshuricum, A. sieboldii var. seoulense, and A. himalaicum by UHPLC-QTOF/MS methods.

\begin{tabular}{|c|c|c|c|c|c|c|}
\hline $\begin{array}{l}\text { Peaks } \\
\text { No. }\end{array}$ & Compounds & $\begin{array}{l}t_{R} \\
(\min )\end{array}$ & $\begin{array}{l}\text { UV } \\
(\mathrm{nm})\end{array}$ & $\begin{array}{l}{[\mathrm{M}+\mathrm{H}]^{+}} \\
(\mathrm{m} / \mathrm{z})\end{array}$ & $\begin{array}{l}\text { Aglycones or diagnos- } \\
\text { tics fragments }(\mathrm{m} / \mathrm{z})\end{array}$ & $\begin{array}{l}\text { Comparison } \\
\text { with standards }\end{array}$ \\
\hline 1 & (2R)-naringenin 5,7-di-O-glucoside & 1.78 & 277,320 & 597.1819 & 435,273 & yes \\
\hline 2 & (2S)-naringenin 5,7-di-O-glucoside & 1.99 & 277,320 & 597.1819 & 435,273 & yes \\
\hline 3 & 1-O-p-coumaroyl-xylopyranosyl-glucoside & 2.00 & 232,315 & 459.1503 & $481,476,165,147$ & yes \\
\hline 4 & 1-O-feruloyl-xylopyranosyl-glucoside & 2.64 & 241,330 & 489.1608 & $511,506,195,177$ & yes \\
\hline 5 & isomer of (2R)-naringenin 5,7-di-O-glucoside & 3.95 & 288,323 & 597.1819 & 435,273 & no \\
\hline 6 & AA-IVa & 6.03 & 242,330 & 358.0563 & 312,297 & yes \\
\hline 7 & 3,4,5-trimethoxytoluene & 6.53 & 269,300 & 183.1021 & 168,153 & no \\
\hline 8 & 2-methoxyl-methylenedioxypropiophenone & 7.52 & 269,332 & 209.0814 & 176,161 & yes \\
\hline 9 & 2,4,6-trimethoxytoluene & 7.52 & 225,280 & 183.1021 & 168,153 & no \\
\hline 10 & kakuol & 8.25 & 277,346 & 195.0657 & 147 & yes \\
\hline 11 & pluviatilol & 9.67 & 232,285 & 357.1338 & 339,289 & yes \\
\hline 12 & methyleugenol & 10.83 & 231,280 & 179.1072 & 164 & yes \\
\hline 13 & AL-I & 10.92 & 239,259 & 294.0766 & n.d. & yes \\
\hline 14 & AA-I & 13.49 & 225,244 & 342.0614 & n.d. & yes \\
\hline 15 & safrole & 15.94 & 235,286 & 163.0759 & n.d. & yes \\
\hline 16 & myristicin & 16.67 & 239,299 & 193.0865 & 192 & yes \\
\hline 17 & sesamin & 16.98 & 241,286 & 355.1182 & $337,319,289$ & yes \\
\hline 18 & $\mathrm{~N}$-isobutyl-2E,4E,8Z-decatetraenamide & 17.30 & 260 & 222.1858 & 167,152 & no \\
\hline 19 & asarinin & 18.09 & 241,286 & 355.1182 & 337,319 & yes \\
\hline 20 & $\mathrm{~N}$-isobutyl-2E,4E,8Z,10Z-dodecatetraenamide & 20.45 & 235,259 & 248.2014 & 167,152 & yes \\
\hline 21 & $N$-isobutyl-2E,4E, $8 Z, 10 E$-dodecatetraenamide & 20.73 & 235,259 & 248.2014 & 167,152 & yes \\
\hline 22 & isomer of $\mathrm{N}$-isobutyl-dodecatetraenamide & 21.48 & 235,259 & 248.2014 & 167,152 & no \\
\hline
\end{tabular}

n. d., not detectable

tively identified as isomer of $\mathrm{N}$-isobutyl-2,4,8,10-dodecatetraenamide.

Peak $18\left(t_{R} 17.30 \mathrm{~min}\right)$ generated the $[\mathrm{M}+\mathrm{H}]^{+}$ions at $\mathrm{m} / \mathrm{z}$ 222.1858, corresponding to the same molecular formula, $\mathrm{C}_{14} \mathrm{H}_{23} \mathrm{NO}$. High collision energy resulted in two diagnostic fragment ions, at $m / z 167.1310$ (loss of $\mathrm{C}_{4} \mathrm{H}_{7}, 55$ mass units) and $\mathrm{m} / \mathrm{z}$ 152.1075 (loss of $\mathrm{C}_{5} \mathrm{H}_{10}, 70$ mass units). The same fragment ions were shown in MS data of peak 20 and 21, suggesting that the compound corresponding to peak $\mathbf{1 8}$ should also be an alkamide. According to the fragmentation pathways in the study by Quang et al. [8], the compound corresponding to peak 18 was tentatively identified as $\mathrm{N}$-isobutyl-2E,4E,8Z-decatetraenamide ( $\mathbf{0}$ Fig. 2).

Good linear calibration curves were obtained with 18 tested reference standards $(R>0.995$, 0 Table 2$)$. Because of a great difference in the contents of 2-methoxyl-4,5-methylene-dioxypropiophenone (8), kakuol (10), and methyleugenol (12) between the 




Fig. 2 Fragmentation pattern and MS spectra of $N$-isobutyl-2E,4E,8Z,10Edodecatetraenamide (peak 21, a) and N-isobutyl-2E,4E,8Z-decatetraenamide (peak $18, \mathbf{b})$.

herbal materials, two calibrations were established to serve for the low and high content levels. The LODs and LOQs were in the range from 0.03 to $24.9 \times 10^{-3} \mu \mathrm{g} / \mathrm{mL}$ and from 0.09 to $82.9 \times 10$ $-3 \mu \mathrm{g} / \mathrm{mL}$, respectively.

The precision and recovery tests were done for 7 major marker compounds by the methods described in Materials and Methods. This method exhibited good reproducibility with intra- and inter-day variations (evaluated with RSD) of less than $4.8 \%$ (Table 2S, Supporting Information). The recovery for these markers ranged from $99.0 \%$ to $103 \%$, with RSD ranging from $2.0 \%$ to $5.8 \%$ (Table 2S, Supporting Information). Thus, the analytical procedure is accurate and sufficiently sensitive for the simultaneous quantification of the major compounds in the raw materials of three Asarum species.

Using the UHPLC-QTOF/MS method, chemical profiling and quantification of the components from the roots and rhizomes of $A$. heterotropoides var. mandshuricum and $A$. sieboldii var. seoulense and the roots of $A$. himalaicum were carried out ( Fig. 1 and Table 3). There is a significant difference in the chemical profiling patterns among the roots and rhizomes of the three Asarum species.

Twenty-two components were commonly found in two raw materials of ARR (A. heterotropoides var. mandshuricum and A. sieboldii var. seoulense), including 5 lignans, 5 essential oils, 3 AAs, 4 alkamides, and 5 flavanoids ( $\$$ Table 3 ).

Asarinin (19) (0.37-3.10 mg/g) and sesamin (17) (0.17-0.73 mg/ g) were the major lignans in the samples of two Asarum species, asarinin (19) being a marker compound for the quality control of ARR according to the Chinese Pharmacopoeia. 2-methoxyl-4,5methylenedioxypropiophenone (8) and kakuol (10) were detected in much greater abundance in the samples of $A$. heterotropoides var. mandshuricum [0.49-1.22 mg/g for 2-methoxyl-4,5methylenedioxypropiophenone (8) and $0.11-0.25 \mathrm{mg} / \mathrm{g}$ for kakuol (10)] than in those of $A$. sieboldii var. seoulense [trace$0.07 \mathrm{mg} / \mathrm{g}$ for 2-methoxyl-4,5-methylenedioxypropiophenone (8) and trace- $0.01 \mathrm{mg} / \mathrm{g}$ for kakuol (10)], suggesting that both characteristic lignans might contribute to chemically distinguish the raw materials of the two Asarum species. Actually, the commercial samples of ARR mostly consist of $A$. heterotropoides var. mandshuricum and A. sieboldii var. seoulense due to their mixed plantation in most herbal farms. According to our results, there is not a relationship between the proportion of the mixed cultivation of different subspecies and the lignan contents. However, the difference in the lignan contents between Asarum heterotropoides var. mandshuricum and A. sieboldii var. seoulense is significant. For example, kakuol (10) and 2-methoxyl-4,5-methylenedioxypropiophenone (8) were detected in much greater abundance in the samples of $A$. heterotropoides var. mandshuricum than in those of $A$. sieboldii var. seoulense, which may serve for the chemical characterization of both Asarum herbal materials. Since the pharmacological studies reported the in vitro and in vivo antifungal activity of both lignans [34], they should be the candidates for standardization of herbal materials of ARR, and the mixed cultivation of the two Asarum plants might not be desirable for the quality control of raw materials of ARR. Zhang et al. [18] reported the determination of three major lignans, asarinin

Table 2 Calibrations and detection limits for marker compounds.

\begin{tabular}{|c|c|c|c|c|c|}
\hline Compounds & Calibration curve & $r$ & $\begin{array}{l}\text { Linear range } \\
(\mu \mathrm{g} / \mathrm{mL})\end{array}$ & $\begin{array}{l}\text { LOD } \\
\left(\times 10^{-3} \mu \mathrm{g} / \mathrm{mL}\right)\end{array}$ & $\begin{array}{l}\text { LOQ } \\
\left(\times 10^{-3} \mu \mathrm{g} / \mathrm{mL}\right)\end{array}$ \\
\hline (2R)-naringenin 5,7-di-O-glucoside (1) & $Y=83.7 x-16.06$ & 1.000 & $0.320-16.00$ & 2.28 & 7.59 \\
\hline (2S)-naringenin 5,7-di-O-glucoside (2) & $Y=99.5 x+19.24$ & 0.999 & $0.170-17.00$ & 2.00 & 6.67 \\
\hline 1-O-p-coumaroyl-xylopyranosyl-glucoside (3) & $Y=55.45 x-2.852$ & 1.000 & $0.200-10.00$ & 3.19 & 10.6 \\
\hline 1-O-feruloyl-xylopyranosyl-glucoside (4) & $Y=256.6 x+42.71$ & 0.998 & $0.050-4.000$ & 0.37 & 1.25 \\
\hline AA-IVa (6) & $Y=186.4 x+4.856$ & 0.999 & $0.050-5.000$ & 0.71 & 2.36 \\
\hline \multirow[t]{2}{*}{ 2-methoxyl-methylenedioxypropiophenone (8) } & $Y=2076 x+667.5$ & 0.999 & $0.400-20.00$ & \multirow[t]{2}{*}{0.03} & \multirow[t]{2}{*}{0.09} \\
\hline & $Y=1721 x+0.4944$ & 0.998 & $0.004-0.400$ & & \\
\hline \multirow[t]{2}{*}{ kakuol (10) } & $Y=818 x+94.4$ & 0.998 & $0.200-10.00$ & \multirow[t]{2}{*}{0.03} & \multirow[t]{2}{*}{0.10} \\
\hline & $Y=1057 x+3.834$ & 0.999 & $0.002-0.200$ & & \\
\hline pluviatilol (11) & $Y=339.7 x-12.55$ & 0.999 & $0.250-10.00$ & 0.89 & 2.96 \\
\hline \multirow[t]{2}{*}{ methyleugenol (12) } & $Y=64.80 x-17.19$ & 0.997 & $5.000-100.0$ & \multirow[t]{2}{*}{0.72} & \multirow[t]{2}{*}{2.41} \\
\hline & $Y=54.29 x-1.018$ & 0.998 & $0.100-5.000$ & & \\
\hline AL-I (13) & $Y=3065 x-82.52$ & 0.998 & $0.020-2.000$ & 0.12 & 0.39 \\
\hline AA-I (14) & $Y=35.86 x+30.49$ & 0.998 & $0.020-2.000$ & 0.03 & 0.14 \\
\hline safrole (15) & $Y=71.31 x+98.2$ & 0.998 & $1.000-10.00$ & 6.12 & 20.4 \\
\hline myristicin (16) & $Y=72.33 x+7.632$ & 0.999 & $0.200-10.00$ & 24.9 & 82.9 \\
\hline sesamin (17) & $Y=358.6 x-103.8$ & 0.999 & $1.000-15.00$ & 0.73 & 2.44 \\
\hline asarinin (19) & $Y=224.3 x+637.8$ & 0.998 & $2.000-50.00$ & 0.44 & 1.47 \\
\hline $\mathrm{N}$-isobutyl-2E,4E,8Z,10Z-dodecatetranamide (20) & $Y=118.8 x-186.5$ & 0.999 & $5.000-100.0$ & 0.26 & 0.88 \\
\hline$N$-isobutyl-2E,4E,8Z,10E-dodecatetranamide (21) & $Y=151.3 x+213.0$ & 0.999 & $2.500-75.00$ & 0.41 & 1.37 \\
\hline Isomer of $\mathrm{N}$-isobutyl-dodecatetranamide (22) & $Y=31.62 x-4.544$ & 0.999 & $0.630-50.00$ & 0.46 & 1.53 \\
\hline
\end{tabular}




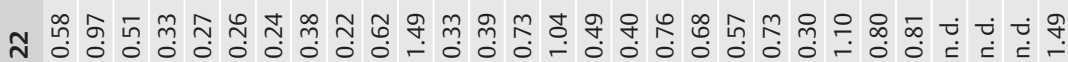

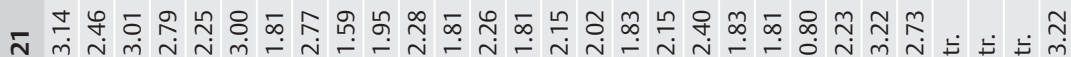

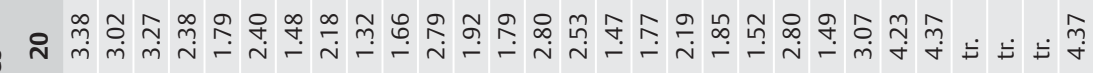
带

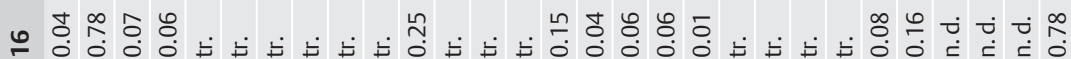
๓ है

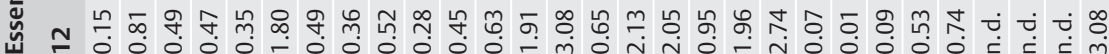

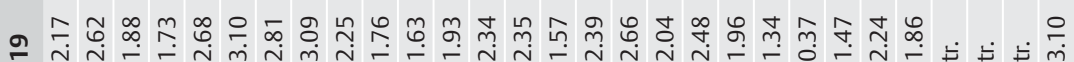

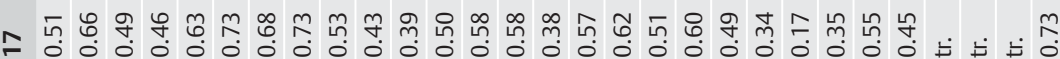

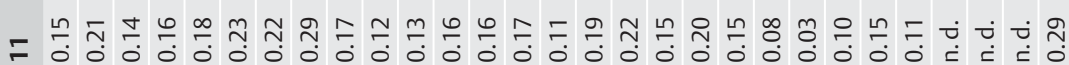

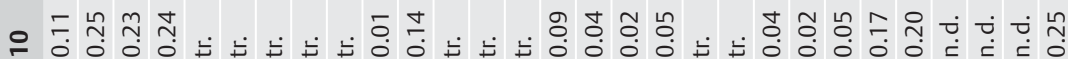

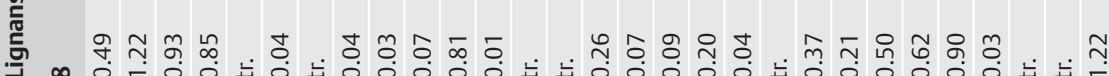

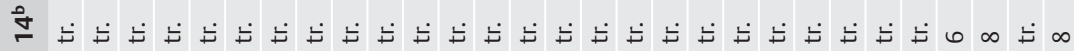

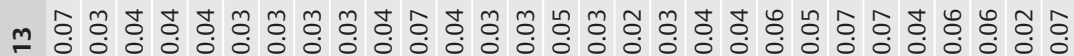
连

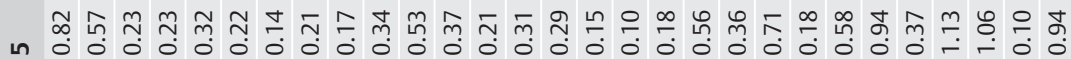

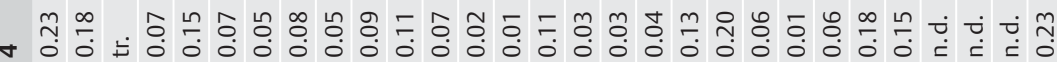

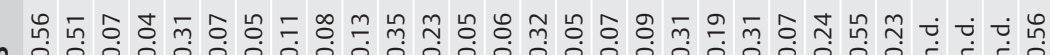
๙ N

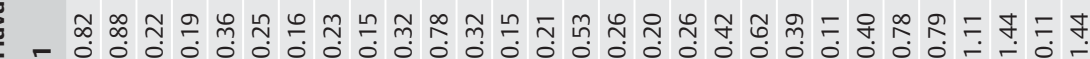


(19), sesamin (17) and kakuol (10), in the roots and rhizomes of ARR by HPLC method, in which the used samples were not authenticated due to their commercial origins. The plant materials can be easily authenticated on the basis of the significant differences in the morphological characteristics of flowers, as described in Materials and Methods.

The chemical profiling of the essential oils of ARR was well studied using the GC-MS method [9]. In the present study, only 3 essential oils, methyleugenol (12), safrole (15), and myristicin (16) were detected and quantified by the UHPLC-UV or UHPLCQTOF/MS methods. Safrole (15), possessing liver carcinogenic effects, was quantified by the UHPLC-UV method because it did not produce mass signal response due to its volatility. Methyleugenol (12), with central inhibition, antibechic and analgetic activity [35, 36], is a marker compound for the quality control of essential oils of the raw materials of ARR and the granules of ARR extract.

The limit test for AAs in ARR has been recorded in the Chinese and Japanese Pharmacopoeias [3,21]. Three AAs in ARR were simultaneously quantified for the first time. AA-I (14) was found in trace amounts (less than $10 \mathrm{ppm}$ ) in all the samples of A. heterotropoides var. mandshuricum and $A$. sieboldii var. seoulense, but with a content of 6-8 ppm in those of $A$. himalaicum. AA-IVa (6) and AL-I (13) were detected in A. heterotropoides var. mandshuricum [0.07-0.16 mg/g for AA-IVa (6) and 0.03$0.07 \mathrm{mg} / \mathrm{g}$ for AL-I (13)] and A. sieboldii var. seoulense [0.03$0.04 \mathrm{mg} / \mathrm{g}$ for AA-IVa (6) and $0.03-0.04 \mathrm{mg} / \mathrm{g}$ for AL-I (13)]. Many papers reported the quantification of AAs in ARR. Sun et al. [37] reported that there is no or just a trace amount of AA-I (14) in the underground parts (namely roots and rhizomes) of $A$. heterotropoides var. mandshuricum and $A$. sieboldii var. seoulense in a small amount of AA-I (14) in their aerial parts. The results from the study of Chen et al. [38] showed that AA-IVa was detected in greater abundance than AA-I (14) in the A. heterotropoides var. mandshuricum roots and rhizomes (26.49-51.73 $\mu \mathrm{g} / \mathrm{g})$. According to our study, AA-IVa (6) and AL-I (13) were found in greater amount in A. heterotropoides var. mandshuricum than in A. sieboldii var. seoulense. Since AA-IVa (6) and AL-I (13) are the potential compounds for side effects of AAN, the planting of $A$. sieboldii var. seoulense should be preferable for GAP of ARR.

Four alkamides were simultaneously quantified or semi-quantified. Among them, $N$-isobutyl-2E,4E,8Z,10Z-dodecatetraenamide (20) and $N$-isobutyl-2E,4E,8Z,10E-dodecatetraenamide (21) were both major alkamides found in the samples of $A$. heterotropoides var. mandshuricum and $A$. sieboldii var. seoulense [1.32-4.37 mg/g for $N$-isobutyl-2E,4E,8Z,10Z-dodecatetraenamide (20) and $0.80-3.22 \mathrm{mg} / \mathrm{g}$ for $N$-isobutyl-2E,4E,8Z,10E-dodecatetraenamide (21)]. Some pharmacological studies indicated that both alkamides showed antioxidant, immunostimulatory, and anti-inflammatory effects $[39,40]$. So they should not be ignored in quality standardization of ARR.

Moreover, 5 flavonoids, (2R)-naringenin 5,7-di- $O$ - $\beta$-D-glucoside (1), (2S)-naringenin 5,7-di-O- $\beta$-D-glucoside (2), 1-O-p-coumaroyl-xylopyranosyl-glucoside (3), 1-O-feruloyl-xylopyranosyl-glucoside (4), and the isomer of (2R)-naringenin 5,7-di- $O$ - $\beta$-D-glucoside (5), were quantified and semi-quantified as minor components in the samples of $A$. heterotropoides var. mandshuricum and A. sieboldii var. seoulense as well the content of all of which were less than $1.0 \mathrm{mg} / \mathrm{g}$.

The root of A. himalaicum is one of the adulterants of ARR in China. There is a significant difference in the chemical composition between the raw materials of $A$. heterotropoides var. mandshuricum and/or A. sieboldii var. seoulense, and those of A. himalaicum.
Twelve components were detected in the roots of $A$. himalaicum, including 3 flavanoids, ( $2 R$ )-naringenin 5,7-di- $O$ - $\beta$-D-glucoside (1), (2S)-naringenin 5,7-di-O- $\beta$-D-glucoside (2), and the isomer of ( $2 R$ )-naringenin 5,7-di- $O-\beta$-D-glucoside (5), 3 AAs, AA-IVa (6), AL-I (13), and AA-I (14), 3 lignans, 2-methoxyl-4,5-methylene-dioxypropiophenone (8), sesamin (17), and asarinin (19), 1 essential oil, safrole (15), and 2 alkamides, $N$-isobutyl$2 E, 4 E, 8 Z, 10 Z$-dodecatetraenamide (20) and $N$-isobutyl-2E,4E, $8 Z, 10 E$-dodecatetraenamide (21). Among them, the 3 flavanoids were the major components of $A$. himalaicum. It is worth noting that AA-I (14) was detected in higher content in A. himalaicum than in the samples of two certified Asarum species in spite of AA-IVa (6) and AL-I (13) being found in similar amounts in the samples of three Asarum species. The results suggested that A. himalaicum should not be used as a medicinal substitute of ARR and should be prohibited in herbal markets in China.

Several studies on HPLC determination of 1-3 marker compounds of ARR have been reported [17-19,37,38], in which asarinin (19), sesamin (17), kakuol (10), methyleugenol (12), safrole (15), AA-I (14), and AA-IVa (6) were involved. The contents of these marker compounds determined by UHPLC-QTOF MS in the present study were similar to those shown in the mentioned above literatures. We established a UHPLC-QTOF/MS profiling and quantification of 5 lignans, 3 AAs, 3 essential oils, 4 alkamides, and 5 flavanoids in $25 \mathrm{~min}$, which may serve for the chemical characterization of the raw materials of the three Asarum plants. Moreover, the established profiles may be more practical for the standardization of the roots and rhizomes of $A$. heterotropoides var. mandshuricum and $A$. sieboldii var. seoulense than the HPLC methods. However, it was difficult to distinguish the mixed commercial samples of ARR using the present chemical profiling. To further visualize the difference between the UHPLC-QTOF/MS profiles obtained from the samples of $A$. heterotropoides var. mandshuricum and A. sieboldii var. seoulense, unsupervised PCA and supervised OPLS-DA were performed to process data and figure out important components for their difference.

The score plot obtained by all observations using 6997 Paretoscaled variables from the two species is displayed in 1 Fig. 3A. A clear separation can be seen between $A$. heterotropoides var. mandshuricum and $A$. sieboldii var. seoulense. $57.9 \%$ of the variables can be explained by two indices, which were calculated by cross validation. The results indicated a significant discrimination between the raw materials of two Asarum species in the profiles. To further find the potential chemical markers for the discrimination between two Asarum samples, 21 marker components (except safrole (15), without MS determination) were screened to perform the OPLS-DA and generate score plot and S-plot ( $\odot$ Fig. 3 B and C). In the present study, R2Y (total explained variation for the X matrix) and Q2 (the predictability of the model) were 0.9909 and 0.9028 , respectively, indicating excellent prediction ability of the model. Thus, the established OPLS-DA model had a good capability to distinguish $A$. heterotropoides var. mandshuricum from $A$. sieboldii var. seoulense. In the S-plot, each point represents an ion $t_{R}-m / z$ pair. The $t_{R}-m / z$ pair points at the two ends of " $\mathrm{S}$ " represent characteristic markers with the most confidence to each group. The VIP (variable importance in the projection) value ensured the significance of potential markers. Five ions, a $\left(t_{R} 17.29 \mathrm{~min}, \mathrm{~m} / \mathrm{z} 222.1856\right.$, VIP 1.67), b $\left(t_{R}\right.$ $8.24 \mathrm{~min}, \mathrm{~m} / \mathrm{z} 195.0660$, VIP 1.63$)$, c $\left(\mathrm{t}_{\mathrm{R}} 7.48 \mathrm{~min}, \mathrm{~m} / \mathrm{z} 209.0812\right.$, VIP 1.63), d ( $t_{R} 6.53 \mathrm{~min}, \mathrm{~m} / \mathrm{z} 183.1022$, VIP 1.51), and e $\left(t_{R}\right.$ $20.48 \mathrm{~min}, \mathrm{~m} / \mathrm{z} 248.2015$, VIP 1.36$)$, at the bottom left corner, and two ions, $\mathrm{f}\left(\mathrm{t}_{\mathrm{R}} 18.08 \mathrm{~min}, \mathrm{~m} / \mathrm{z} 353.1180\right.$, VIP 1.52$)$ and $\mathrm{g}\left(\mathrm{t}_{\mathrm{R}}\right.$ 

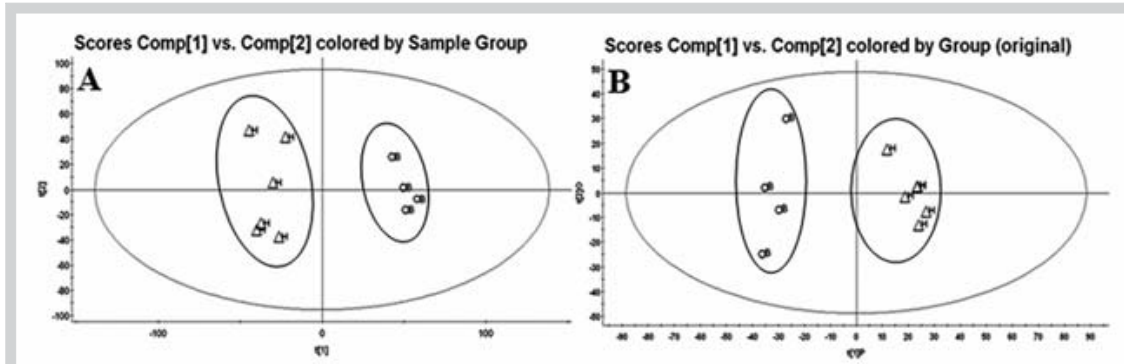

Fig. 3 PCA scores plot (A), OPLS-DA Plot (B), and S-plot (C) of four $A$. heterotropoides var. mandshuricum and six $A$. sieboldii var. seoulense samples. Circles and open triangles in (A) and (B) represent A. heterotropoides var. mandshuricum and $A$. sieboldii var. seoulense, respectively. The points $\mathbf{a} \sim \mathbf{g}$ in the $\mathrm{S}$ plot $(\mathbf{C})$ represent the 7 seven leading markers.

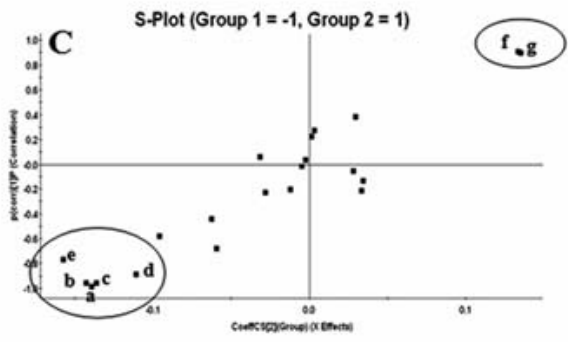

$16.97 \mathrm{~min}, \mathrm{~m} / \mathrm{z} 353.1184$, VIP 1.51 ), at the top right corner of " $\mathrm{S}$ ", are the marker compounds of $A$. heterotropoides var. mandshuricum and $A$. sieboldii var. seoulense, respectively, which contribute most to the difference between the raw materials of the two Asarum species.

The 7 leading markers between the raw materials of $A$. heterotropoides var. mandshuricum and/or $A$. sieboldii var. seoulense were structurally identified as $N$-isobutyl-(2E,4E,8Z)-decatrienamide (18) (a), kakuol (10) (b), 2-methoxyl-4,5-methylene-dioxypropiophenone (8) (c), 3,4,5-trimethoxytoluene (7) (d), $N$-isobutyl$2 E, 4 E, 8 Z, 10 E$-dodecatetraenamide (21) (e), asarinin (19) (f), and sesamin (17) (g), respectively. The results of multivariate statistical analysis supported the quantification results in our study.

In conclusion, the present study is the first report on a UHPLCQTOF/MS method for the rapid structural elucidation of 5 lignans, 5 essential oils, 3 AAs, 4 alkamides, and 5 flavanoids from the roots and rhizomes of $A$. heterotropoides var. mandshuricum, A. sieboldii var. seoulense and the roots of A. himalaicum. Moreover, the method was validated and applied for simultaneous quantification and semi-quantification of 20 compounds among them with good accuracy and precision. The difference in the chemical profiles among the samples of three Asarum species is helpful for the standardization and quality control of plant materials of ARR. Furthermore, the determined markers are representative according to multivariate statistical analysis.

\section{Materials and Methods}

$\nabla$

\section{Chemicals and reagents}

Eighteen reference compounds were used in the present study (Fig. 1S, Supporting Information). Methyleugenol (12), AL-I (13), and AA-I (14) were purchased from National Institutes for Food and Drug Control (China), and AA-IVa (6), kakuol (10), safrole (15), sesamin (17) and asarinin (19) were from Shanghai Forever Biotech Co., Ltd. Other reference compounds used were isolated from the extracts of the roots and rhizomes of $A$. heterotropoides var. mandshuricum and $A$. sieboldii var. seoulense in our previous studies $[41,42]$. They included $(2 R)$-naringenin $5,7-d i-O-\beta$-D-glu- copyranoside (1), (2S)-naringenin 5,7-di- 0 - $\beta$-D-glucopyranoside (2), 1-O-p-coumaroyl- $\beta$ - $D$-xylopyranosyl- $(1 \rightarrow 6)-\beta$ - $D$-glucopyraboside (3), 1-O-feruloyl- $\beta$ - $D$-xylopyranosyl-( $1 \rightarrow 6)-\beta$ - $D$-glucopyraboside (4), 2-methoxyl-4,5-methylene-dioxypropiophenone (8), pluviatilol (11), and myristicin (16). The identity of these compounds was confirmed by melting point, UV, IR, ${ }^{1} \mathrm{H}-$ and ${ }^{13} \mathrm{C}-\mathrm{NMR}$, and MS, and their purities evaluated with HPLC-DAD were more than $98 \%$. Two standards, $N$-isobutyl-2E,4E,8Z,10Zdodecatetraenamide (20) and $N$-isobutyl-2E,4E,8Z,10E-dodecatetraenamide (21), were isolated from Echinacea purpurea in our previous study [43], and their structures were confirmed by melting point, UV, IR, ${ }^{1} \mathrm{H}$ - and ${ }^{13} \mathrm{C}-\mathrm{NMR}$, MS and compared with the literature. The standard of an isomer of $\mathrm{N}$-isobutyl-2,4,8,10dodecatetraenamide (22) was isolated from the extracts of the roots and rhizomes of $A$. heterotropoides var. mandshuricum and A. sieboldii var. seoulense, and its structure was tentatively identified by the UV spectra, MS/MS determination along with Waters MassFragment software, and comparison with literature [10]. However, the configurations of $\mathrm{C}_{2}, \mathrm{C}_{4}, \mathrm{C}_{8}$, and $\mathrm{C}_{10}$ could not be determined by MS/MS. The purities of the three compounds evaluated with HPLC-DAD were more than 98\%. ACN (HPLC-MS grade) and formic acid (spectroscopy grade) were purchased from Fisher Scientific UK. Ultrapure water (18.2 M $\Omega$ ) was daily prepared with a Milli-Q water purification system (Millipore). Leucine-enkephalin was obtained from Sigma-Aldrich.

\section{Plant materials}

Twenty-seven samples (samples \#1 \#27) were directly obtained from China (Table 1S, Supporting Information). The plant materials were authenticated by Prof. Weining Wang (Liaoning Institute for Food and Drug Control, China). They were identified as $A$. heterotropoides var. mandshuricum or A. sieboldii var. seoulense according to the shape of the perianth lobes and leaves [44]. The perianth lobe of $A$. heterotropoides var. mandshuricum is erect with acuminate leaf blade apex, while that of $A$. sieboldii var. seoulense curls with acute leaf blade apex. We could only authenticate some samples according to the phytomorphology of the flowers in the fresh materials, while others from herbal markets were recorded as "Liaoxixin" according to their commercial 
names and the general appearance identification. The voucher specimens are kept in the reference library for the medicinal herbs in Shenyang Pharmaceutical University. For voucher specimens numbers and collection details, see Table 1S Supporting Information.

\section{Instrumentation and chromatographic conditions}

UHPLC analysis was performed on a Waters Acquity $\mathrm{HSSC}_{18}$ column $(2.1 \times 100 \mathrm{~mm}, 1.8 \mu \mathrm{m}$, Waters $)$ at $40^{\circ} \mathrm{C}$. The mobile phase consisted of (A) water containing $0.2 \%$ formic acid and (B) acetonitrile containing $0.2 \%$ formic acid with gradient elution (linear gradient $9 \%$ B in 2.5 min, linear gradient $9 \%$ B to $31 \%$ B between 2.5 and $4 \mathrm{~min}$, linear gradient $31 \% \mathrm{~B}$ between 4 and $15 \mathrm{~min}$, linear gradient $31 \%$ B to $40 \%$ B between 15 and 16 min, linear gradient $40 \%$ B to $44 \%$ B between 16 and 17.5 min, linear gradient $40 \%$ B to $44 \%$ B between 16 and 17.5 min, finally linear gradient $44 \%$ B between 17.5 and $21.5 \mathrm{~min}$ ). Re-equilibration duration (linear gradient $44 \%$ to $99 \%$ B between $21.5 \mathrm{~min}$ and $22 \mathrm{~min}$, linear gradient 99\% B between 22 and 23 min, linear gradient 99\% B to 9\% B between 23 and $23.1 \mathrm{~min}$, finally $9 \%$ B between 23.1 and $25 \mathrm{~min}$ ) was $2.5 \mathrm{~min}$ between individual runs. The flow rate was kept at $0.6 \mathrm{~mL} / \mathrm{min}$, and $2 \mu \mathrm{L}$ of standard and sample solution were injected in each run.

Identification of marker compounds by UHPLC-QTOF/MS was performed on Waters QTOF Xevo G2 equipped with an ESI source, which gives a resolution of 10000 (FWHM) and mass accuracy error less than $5 \mathrm{ppm}$. Leucine-enkephalin was used as the lock mass to generate an $[\mathrm{M}+\mathrm{H}]^{+}$ion $(m / z 556.2771)$ in the LockSpray mode at a concentration of $50 \mathrm{pg} / \mu \mathrm{L}$ at an infusion flow rate of $10 \mu \mathrm{L} / \mathrm{min}$. The ESI source was operated in positive ionization mode with the capillary voltage at $3.0 \mathrm{kV}$, and the cone voltage was set to $25 \mathrm{~V}$. Source and desolvation temperatures were set at 130 and $450^{\circ} \mathrm{C}$, respectively. The nebulization gas flows were $800 \mathrm{~L} / \mathrm{h}$. All data collected in centroid mode were acquired using Masslynx ${ }^{\mathrm{TM}}$ NT 4.1 software (Waters Corp.).

Two different MS scanning experiments were used. (1) MSE experiment ( $E$ represents collision energy) uses an intelligent approach where parallel alternating scans are acquired both at low-collision and high-collision energy to obtain precursor ion information and full-scan mass fragment with precursor ion information in a single analytical run, respectively. The MSE experiment in two scan functions was carried out as follows. Function 1: $m / z$ 100-1200, 0.2 s scan time, 6 V collision energy; and function 2: $m / z$ 100-1200, 0.2 s scan time, collision energy ramp of 20-30 V. (2) MS/MS experiments were carried out by ramping collision energies from 20 and $30 \mathrm{~V}$.

\section{Preparation of standard solutions}

Seventeen reference compounds, including ( $2 R$ )-naringenin 5,7di- $O$ - $\beta$-D-glucopyranoside (1), (2S)-naringenin $5,7-\mathrm{di}-O-\beta$-Dglucopyranoside (2), 1-O-p-coumaroyl- $\beta$ - $D$-xylopyranosyl- $(1 \rightarrow$ $6)-\beta$ - $D$-glucopyraboside (3), 1-O-feruloyl- $\beta$ - $D$-xylopyranosyl$(1 \rightarrow 6)-\beta$ - $D$-glucopyraboside (4), AA-IVa (6) 2-methoxyl-4,5methylene-dioxypropiophenone (8), kakuol (10), pluviatilol (11), methyleugenol (12), AL-I (13), AA-I (14), safrole (15), myristicin (16), sesamin (17), asarinin (19), $N$-isobutyl-2E,4E,8Z,10Zdodecatetraenamide (20), and $N$-isobutyl-2E,4E,8Z,10E-dodecatetraenamide (21), as well as one tentatively identified compound, the isomer of $\mathrm{N}$-isobutyl-2,4,8,10-dodecatetraenamide (22), were accurately weighed and dissolved in methanol to give individual stock solutions at suitable concentration. Series of working standard solutions were prepared by appropriate dilu- tion of the stock solution with methanol in order to prepare calibrators. All solutions were stored at $4{ }^{\circ} \mathrm{C}$ in refrigerator before analysis.

\section{Sample preparation}

Powdered herbal materials ( $0.5 \mathrm{~g}$, passed through a $500 \mu \mathrm{m}$ mesh sieve) were extracted by reflux with $50 \mathrm{~mL}$ of methanol for $30 \mathrm{~min}$. The mixtures were centrifuged at $3000 \mathrm{rpm}$ for $5 \mathrm{~min}$, the supernatants were evaporated to dry under vacuum at $35^{\circ} \mathrm{C}$, and the residues were dissolved with methanol, transformed to a $5 \mathrm{~mL}$ of volumetric flask and diluted with methanol to volume. An aliquot of each $2 \mu \mathrm{L}$ filtrate filtered through a $0.22 \mu \mathrm{m}$ PTFE syringe filter (Whatman, MN, Nal-gene, Advantec) was injected into the UHPLC instrument for analysis.

\section{Method validation for quantification}

Among 22 identified compounds, 18 compounds were quantified on an UHPLC-UV or UHPLC-QTOF/MS. Safrole (16) was determined at the detective wavelength of $287 \mathrm{~nm}$ by UHPLC-UV method due to no response in the MS chromatogram. Twelve compounds $(3,4,6,8,10-15,17$, and 19) were quantified using quasi-molecular ion chromatograms (XICs, with a $0.02 \mathrm{Da}$ window), all peak areas of which were integrated at the expected retention times under full-scan MS conditions (Waters QuanLynx ${ }^{\mathrm{TM}}$ version 4.0 software). Five compounds (1, 2, and 20-22) were quantified using quasi-molecular ion chromatograms, all peak areas of which were integrated at the expected retention times under MS/MS conditions at $m / z 435.1291$ ( 1 and 2) and $m / z$ 167.1310 (20-22). Due to lack of standards to complete the validation procedure, the calibration curves for peaks $\mathbf{5}$ and $\mathbf{1 8}$ were not established. Their contents were calculated by the method of semi-quantification with the calibrations of peaks 1 and 22, respectively, because peak 5 showed the same aglycone ion at $\mathrm{m} / \mathrm{z}$ 435.1291 as peak $\mathbf{1}$, and peak $\mathbf{2 2}$ showed the same fragment ion at $m / z 167.1310$ as peak 18. A similar assumption was made by Liu et al. [45].

\section{Calibration curves}

Calibration curves (5-point) were obtained using external standard calibrations for 18 analytes injecting each solution in triplicates and then constructed by plotting the peak area versus the concentration of each analyte.

\section{Limit of detection and of quantitation}

The stock solutions of 18 reference compounds were diluted to a range from 0.03 to $24.9 \times 10^{-3} \mu \mathrm{g} / \mathrm{mL}$, and the injection volume was $2 \mu \mathrm{L}$. LOD and LOQ were determined at a signal-to-noise ratio $(\mathrm{S} / \mathrm{N})$ of about 3 and 10 , respectively.

Precision, accuracy, repeatability, stability, and recovery The intra- and inter-day precisions were evaluated by analyzing known concentrations of the 7 analytes, including 1-O-p-coumaroyl- $\beta$ - $D$-xylopyranosyl-( $1 \rightarrow 6)-\beta$ - $D$-glucopyraboside (3), 2 methoxyl-4,5-methylene-dioxypropiophenone (8), methyleugenol (12), AL-I (13), safrole (16), asarinin (19), and $N$-isobutyl$2 E, 4 E, 8 Z, 10 E$-dodecatetraenamide (21), in six replicates during a single day and by duplicating the experiments on 3 successive days. Six different sample solutions prepared from the same sample were analyzed to confirm the repeatability of the developed assay. Stability of sample solutions was analyzed at $0,2,4,8,12$ and $24 \mathrm{~h}$ at room temperature, respectively. Variations were expressed by RSD. 
The recovery was used to evaluate the accuracy of the method. A known amount of the 7 standards mixed solutions were added into a certain amount of the samples of "Liaoxixin" (A. heterotropoides var. mandshuricum and A. sieboldii var. seoulense) $(0.25 \mathrm{~g}$, sample \#17). The mixture was extracted and analyzed using the method mentioned above. Three replicates were performed for the test.

\section{Chemometric data analysis}

The UHPLC-MS data of A. heterotropoides var. mandshuricum and A. sieboldii var. seoulense samples were analyzed by MarkerLynx XS software (Waters). The parameters were set as following: retention time range 1.5-22.0 min; mass range $\mathrm{m} / \mathrm{z} 100-600 \mathrm{Da}$; retention time tolerance $0.1 \mathrm{~min}$; mass tolerance $0.05 \mathrm{Da}$; width of an average peak at $5 \%$ height and peak-to-peak baseline noise were automatically calculated; marker intensity threshold 10.0; noise elimination level 6.0; isotopic peaks were excluded for analysis.

\section{Supporting information}

The chemical structures of the analysed compounds, collection data of the herbal material samples used in the study, as well as intra-day, inter-day precision and recovery of the seven major marker compounds are available as Supporting Information.

\section{Acknowledgments}

This work was supported by the National Science and Technology Support Program during the Twelfth Five-Year Plan of China (2011BAI03B05), the Distinguished Professor Foundation of Liaoning Province of China of 2011, and the Innovative Drug Incubation Base Plan Project 2013 from the Liaoning Province of China (2013226027).

\section{Conflict of Interest}

\section{$\nabla$}

There are no financial/commercial conflicts of interest involving any of the authors of this study.

\section{References}

1 Drew AK, Whyte IM, Bensoussan A, Dawson AH, Zhu X, Myers SP. Chinese herbal medicine toxicology database: monograph on herba asari, "Xi Xin". Clin Toxicol 2002; 40: 169-172

2 Kim SJ, Zhang CG, Lim JT. Mechanism of anti-nociceptive effects of Asarum sieboldii Miq. Radix: potential role of bradykinin, histamine and opioid receptor-mediated pathways. J Ethnopharmacol 2003; 88: 5-9

3 Chinese Pharmacopoeia Commission. Pharmacopoeia of the People's Republic of China, Volume I. Beijing: Chemical Industry Press; 2010: 214-215

4 Kosuge T, Yokota M, Nukaya H, Gotoh Y, Nagasawa M. Studies on antitussive principles of Asiasari radix. Chem Pharm Bull 1978; 26: 22842285

5 Dan Y, Liu HY, Gao WW, Chen SL. Activities of essential oils from Asarum heterotropoies var. mandshurium against five phytopathogens. Crop Prot 2010; 29: 295-299

6 Lee JY, Moon SS, Hwang BK. Isolation and antifungal activity of kakuol, a propiophenone derivative from Asarum sieboldii rhizome. Pest Manag Sci 2005; 61: 821-825

7 Baluchnejadmojarad T, Roghani M, Nadoushan MRJ, Mahdavi MRV, Kalalian-Moghaddam H, Roghani-Dehkordi F, Dariani S, Raoufi S. The sesame lignan sesamin attenuates vascular dysfunction in streptozotocin diabetic rats: Involvement of nitric oxide and oxidative stress. Eur J Pharmacol 2013; 698: 316-321
8 Quang TH, Ngan NTT, Minh CV, Kiem PV, Tai BH, Thao NP, Song SB, Kim $Y H$. Anti-inflammatory and PPAR transactivational effects of secondary metabolites from the roots of Asarum sieboldii. Bioorg Med Chem Lett 2012; 22: 2527-2533

9 Li C, Xu F, Cao C, Shang MY, Zhang CY, Yu J, Liu GX, Wang X, Cai SQ. Comparative analysis of two species of Asari Radix et Rhizoma by electronic nose, head space GC-MS and chemometrics. J Pharmaceut Biomed 2013; 2: 231-238

10 Yasuda I, Takeya K, Itokawa $H$. Structures of amides from Asiasarum heterotropoides Maek. var. mandshuricum Maek. Chem Pharm Bull 1981; 29: 564-566

11 Han AR, Kim HJ, Shin M, Hong M, Kim YS, Bae H. Constituents of Asarum sieboldii with inhibitory activity on lipopolysaccharide (LPS)-induced NO production in BV-2 microglial cells. Chem Biodivers 2008; 5: 346351

12 Cai SQ Wang X, Zhu S, Li JS, Lou ZQ Namba T, Komatsu K. Studies on botanical origin of commercial samples of traditional Chinese drug Xixin (Herba Asari). Chin J Chin Mater Med 1996; 21: 712-717, 760

13 Li YL, Yu J, Cao C, Xie BB, Zhang CY, Liu Z, Shang MY, Wang X, Cai SQ. Survey on resources and varietie on Chinese markets of crude drug Xixin. Chin J Chin Mater Med 2010; 35: 3237-3241

14 Cai SQ Yu J, Wang X, Wang RQ Ran FX, Shang MY, Cui JR, Komatsu K, Namba T. Cytotoxic activity of some Asarum plants. Fitoterapia 2008; 79: 293-297

15 Xie BB, Shang MY, Wang X, Cai SQ Lee KH. A new aristolochic acid derivative from Asarum himalaicum. Acta Pharm Sin 2011; 46: 188-192

16 Xie YY, Qu JL, Wang QL, Wang Y, Yoshikawa M, Yuan D. Comparative evaluation of cultivars of Chrysanthemum morifolium flowers by HPLC-DAD-ESI/MS analysis and antiallergic assay. J Agric Food Chem 2012; 60: 12574-12583

17 Wang GF, Zhang SR, Zhu SQ. Determination of L-sesamin and L-asarinin in Asarum heterotropoides Fr. Schmidt var. mandshuricum (Maxim.) Kitag. by HPLC. Chin J Pharm Anal 1999; 19: 251-253

18 Zhang L, Chen XH, Liu YL, Zhang J, Bi KS. Simultaneous determination of L-asarinin, L-sesamin and kakuol in Asarum heterotropoides by RPHPLC. Chin Tradit Herbal Drugs 2008; 39: 1098-1100

19 Liu YH, Yi JH, Huang J, Huang ZF, Liu YH. RP-HPLC simultaneous determination of methyleugenol, safrole and asarinin in Asari Radix et Rhizoma. Chin J Pharm Anal 2012; 32: 822-825

20 Zhao ZZ, Liang ZT, Jiang ZH, Leung KSY, Chan CL, Chan HY, Sin J, Man TO, Law KW. Comparative study on the aristolochic acid I content of Herba Asari for safe use. Phytomedicine 2008; 15: 741-748

21 The Ministry of Health, Labour and Welfare. Japanese Pharmacopeia 16th edition. Tokyo: The Ministry of Health, Labour and Welfare; 2011: 1602-1603

22 Hashimoto K, Higuchi M, Makino B, Sakakibara I, Kubo M, Komatsu Y, Maruno M, Okada M. Quantitative analysis of aristolochic acids, toxic compounds, contained in some medicinal plants. J Ethnopharmacol 1999; 64: 185-189

23 Jong TT, Lee MR, Hsiao SS, Hsai JL, Wu TS, Chiang ST, Cai SQ. Analysis of aristolochic acid in nine sources of Xixin, a traditional Chinese medicine, by liquid chromatography/atmospheric pressure chemical ionization/tandem mass spectrometry. J Pharmaceut Biomed 2003; 33: 831837

24 Yuan J, Liu Q Zhu W, Ding L, Tang F, Yao S. Simultaneous analysis of six aristolochic acids and five aristolactams in herbal plants and their preparations by high-performance liquid chromatography-diode array detection-fluorescence detection. J Chromatogr A 2008; 1182: 85-92

25 Li J, Zhang L, Jiang ZZ, Shu B, Li F, Bao Q Zhang LY. Toxicities of aristolochic acid I and aristololactam I in cultured renal epithelial cells. Toxicol In Vitro 2012; 24: 1092-1097

26 Balachandran P, Wei F, Lin RC, Khan IA, Pasco DS. Structure activity relationships of aristolochic acid analogues: Toxicity in cultured renal epithelial cells. Kidney Int 2005; 67: 1797-1805

27 Sato N, Takahashi D, Chen SM, Tsuchiya R, Mukoyama T, Yamagata SI, Ogawa M, Yoshida M, Kondo S, Satoh N, Ueda S. Acute nephrotoxicity of aristolochic acids in mice. J Pharm Pharmacol 2004; 56: 221-229

28 Tistaert C, Dajaegher B, Heyden YV. Chromatographic separation techniques and handing methods for herbal fingerprints: A review. Anal Chim Acta 2011; 902: 148-161

29 Zhang A, Sun H, Wang P, Han Y, Wang X. Modern analytical techniques in metabolomics analysis. Analyst 2012; 137: 293-300

30 Yamamoto H, Kuribayashi H, Seshima Y, Zhao P, Kouno I, Taguchi G, Shimomura K. Metabolism of administered (2RS)-naringenin in flavonoid- 
producing cultured cells of Sophora flavescens. Plant Biotechnol 2004; 21: $355-359$

31 Chen JW, Wu LL, Li X, Zhou HY, Wu TZ. GC-MS analysis of volatile components in supercritical fluid extracts of the root and rhizome of Asarum heterotropoides Fr. var. mandshuricum. Nat Prod Res Dev 2012; 24 : 195-198

32 Bijak M, Saluk J, Tsirigotis-Maniecka M, Komorowska H, Wachowicz B, Zacynska E, Czarny A, Czechowski F, Nowak P, Pawlaczyk I. The influence of conjugates isolated from Matricaria chamomilla L. on platelets activity and cytotoxicity. Int J Biol Macromol 2013; 61: 218-229

33 Luo XB, Chen B, Yao SZ, ZengJG. Simultaneous analysis of caffeic acid derivatives and alkamides in roots and extracts of Echinacea purpurea by high-performance liquid chromatography-photodiode array detection-electrospray mass spectrometry. J Chromatogr A 2003; 986: 7381

34 Zhang $Y$, Shi W, Xi T, Zhang Y, Zhou W. Synthesis and antifungal activity of 1-(2-acyloxy-4, 5-methylenedioxy)phenyl-2-methyl propylene ketones. Chin J Pestic Sci 2012; 14: 95-98

35 Jiang Y, Liu GQ Ma JR, Xie L, Wu HQ. The pharmacological studies on methyleugenol. Acta Pharm Sin 1982; 17: 87-92

36 Zhou HQ Yu B, Qiao WH, Wang DY, Zhang WR, Bai Y, Su YM. Research on pharmacological activities of methyleugenol. Acta Chin Med Pharm 2000; 28: 79-80

37 Sun $\mathrm{Q} W u$ Y, Jia L. Quantitative Determination of Aristolochic Acid in Asarum heterotropoides Fr. Schmidt var. mandshuricum (Maxim.) Kitag. Asian J Tradit Med 2006; 1: 5-13
38 Chen J, Sun BH, Huang J, Gao HY, Wang JQ Wu LJ. Determination of aristolochic acid IVA in the different part of Asarum heterotropoides Fr. Schmidt var. mandshuricum (Maxim.) Kitag. by HPLC. J Shenyang Pharm Univ 2011; 28: 797-800

39 Goey AKL, Rosing H, Meijerman I, Sparidans RW, Schellens JHM, Beijnen $J \mathrm{H}$. The bioanalysis of the major Echinacea purpurea constituents dodeca-2E,4E,8Z,10E/Z-tetraenoic acid isobutylamides in human plasma using LC-MS/MS. J Chromatogr B 2012; 902: 151-156

40 Gallo M, Ferracane R, Naviglio D. Antioxidant addition to prevent lipid and protein oxidation in chicken meat mixed with supercritical extracts of Echinacea angustifolia. J Supercrit Fluid 2012; 72: 198-204

$41 \mathrm{Lv}$ S, Xu L, Huang J, Gao HY, Wu LJ. Chemical constituents of the root of Asarum heterotropoides Fr. Schmidt var. mandshuricum (Maxim.) Kitag. J Shenyang Pharm Univ 2008; 25: 702-704

42 Qi W, Wang LL, Wen H, Zhang L, Yuan D. Isolation and identification of chemical constituents in roots and rhizomes of Asarum heterotropoides Fr. Schmidt var. mandshuricum (Maxim.) Kitag. J Shenyang Pharm Univ 2014; 31: 681-686

43 Kong L. Studies on quality evaluation of Chinese Echinacea purpurea [master]. Shenyang: Shenyang Pharmaceutical University; 2006

44 Delectis Florae Reipublicae Popularis Sinicae Agendae Academiae Sinicae Edita. Flora Reipublicae Popularis Sinicae, Tomus 24. Beijing: Science Press; 1988: 176-177

45 Liu Q Shi Y, Wang Y, Lu J, Cong W, Luo G, Wang Y. Metabolism profile of scutellarin in urine following oral administration to rats by ultra performance liquid chromatography coupled to time-of-flight mass spectrometry. Talanta 2009; 80: 84-91 